\title{
Inflammatory Response in Enterocolitis in the Piebald Lethal Mouse Model of Hirschsprung's Disease
}

\author{
T. FUJIMOTO, D. J. REEN, AND P. PURI \\ Children's Research Centre, Our Lady's Hospital for Sick Children, Crumlin, Dublin, Ireland
}

\begin{abstract}
Mucosal immune defense responses in two clinical forms of enterocolitis complicating congenital megacolon in the piebald lethal mouse model of Hirschsprung's disease were investigated. Significant histological and immunocytochemical differences were seen in the ganglionic segment of colon between piebald mice with early clinical onset of acute illness and piebald mice with late onset enterocolitis. The number of IgA-containing plasma cells infiltrating the lamina propria of the gut in the former group were significantly increased $(p<0.001)$ compared to the latter group of mice and healthy controls. This study suggests that the two forms of enterocolitis complicating congenital megacolon in piebald mice have different etiologies. (Pediatr Res 24: 152-155, 1988)
\end{abstract}

Enterocolitis remains the most serious complication of Hirschsprung's disease resulting in high morbidity and mortality. The reported incidence of enterocolitis ranges from $20-58 \%$ (14). Whereas the cause of enterocolitis is unknown, a number of factors have been implicated in the pathogenesis of this disorder. These include mechanical dilatation and fecal stasis (3), alteration in mucin components (5), occurrence of Schwartzman type reaction (6), increased prostaglandin $E 1$ activity (7), and more recently Clostridium difficile (two phases) infection (8). The possibility that microbial cell host interaction in the gut is involved in the pathogenesis of enterocolitis led us to examine the nature of the local inflammatory response in an animal model of Hirschsprung's disease.

The piebald lethal strain of mice is the most extensively studied animal model for Hirschsprung's disease (9-11). Congenital megacolon in this mouse model is an inherited trait that results in aganglionosis of the terminal segment of the large intestine and development of fecal stasis and megacolon. We have recently identified two clinical forms of enterocolitis in this mouse model, resulting in two distinct patterns of mortality (12). Whereas twothirds of mice with congenital megacolon become acutely ill with severe enterocolitis and die at 3-4 wk of age, the remainder develop massive abdominal distension and classical megacolon and die between 9 and 11 wk of age. The aim of our study was to investigate mucosal defense mechanisms in these two clinical forms of enterocolitis complicating congenital megacolon in this murine model.

\section{MATERIALS AND METHODS}

Animals. A breeding colony of piebald lethal mice was established from a small number of heterozygotes and homozygotes

Received December 8, 1987; accepted March 15, 1988.

Correspondence and requests for reprints Mr. Prem Puri, F.A.C.S., Children's Research Centre, Our Lady's Hospital for Sick Children, Crumlin, Dublin 12, Ireland. obtained from the Jackson Laboratory, Bar Harbour, Maine. The heterozygotes $(\mathrm{SL} /+)$ and homozygous dominants $(+/+)$ of the strain have a dark-colored coat, but the recessive (SL/SL) can be easily distinguished from about 2 days old by their white coat with dark patches. Three groups of mice were used in the study. Group I consisted of seven piebald lethal mice (SL/SL) who became critically sick at 3-4 wk of age and clinically had signs and symptoms of enterocolitis such as diarrhea, anorexia, weight loss, lethargy, and shivering. Group II consisted of seven piebald lethal mice (SL/SL) who became sick at 9-11 wk of age and had diarrhea and massive abdominal distention. Group III consisted of seven piebald mice $(+/+)$ which were control normal litter mates 4-5 wk old.

Tissue preparation. Piebald mice in group I and II were killed at the time of acute illness. The distal, middle, and proximal segments of large bowel corresponding to the constricted aganglionic segment, dilated megacolon segment, and normal colon segment, respectively, were removed from the piebald lethal mice (SL/SL). Colon from control mice was divided into proximal and distal segments. Colonic tissue was immersed for $24 \mathrm{~h}$ in cold $0.01 \mathrm{M}$ phosphate-buffer saline ( $\mathrm{pH} 7.6$ ) to remove extracellular molecular components and then fixed in $96 \%$ cold ethanol at $4^{\circ} \mathrm{C}$ for $12 \mathrm{~h}$ followed by a routine paraffin embedding technique (13). Tissue blocks containing the entire distal, middle, and proximal segments of the large bowel from 21 mice were prepared and kept at $4^{\circ} \mathrm{C}$ until sections were cut.

Hematoxylin and eosin staining. Five- $\mu$ thick sections were cut from all tissue blocks and first screened by conventional hematoxylin and eosin staining.

Immunocytochemistry. Five- $\mu$ thick serial sections of colon were mounted on glass slides, deparaffinated in xylene, and passed from absolute alcohol to water and then immersed in $0.1 \%$ trypsin in $0.1 \% \mathrm{CaCl}_{2}$ solution at $37^{\circ} \mathrm{C}$ for $30 \mathrm{~min}$ before application of first layer antibody. Immunocytochemical studies were carried out by the indirect immunofluorescence method of Coons (10) with immunoglobulin isotype-specific goat antimouse IgA, IgM, and IgG antibodies (N.L. Cappel Laboratories, Cochranville, PA). Positively staining cells were visualized on a Leitz epifluorescence microscope using FITC-labeled rabbit antigoat antibody (Cappel). Cells staining for $\operatorname{IgA}$, IgM, or IgG were counted along the entire colon in three visual fields in each of 10 serial sections from all blocks and expressed as number of cell $/ \mathrm{mm}_{2}$ using a calibrated eye-piece graticule.

Statistics. Statistical analysis was performed using WilcoxonRank sum method.

\section{RESULTS}

The gross appearances in group I piebald mice consisted of a continuous column of feces, above an empty constricted rectosigmoid segment. The spleen was found to be enlarged in all animals in this group. In group II mice, the midregion of the large intestine was distended to about four times the normal 


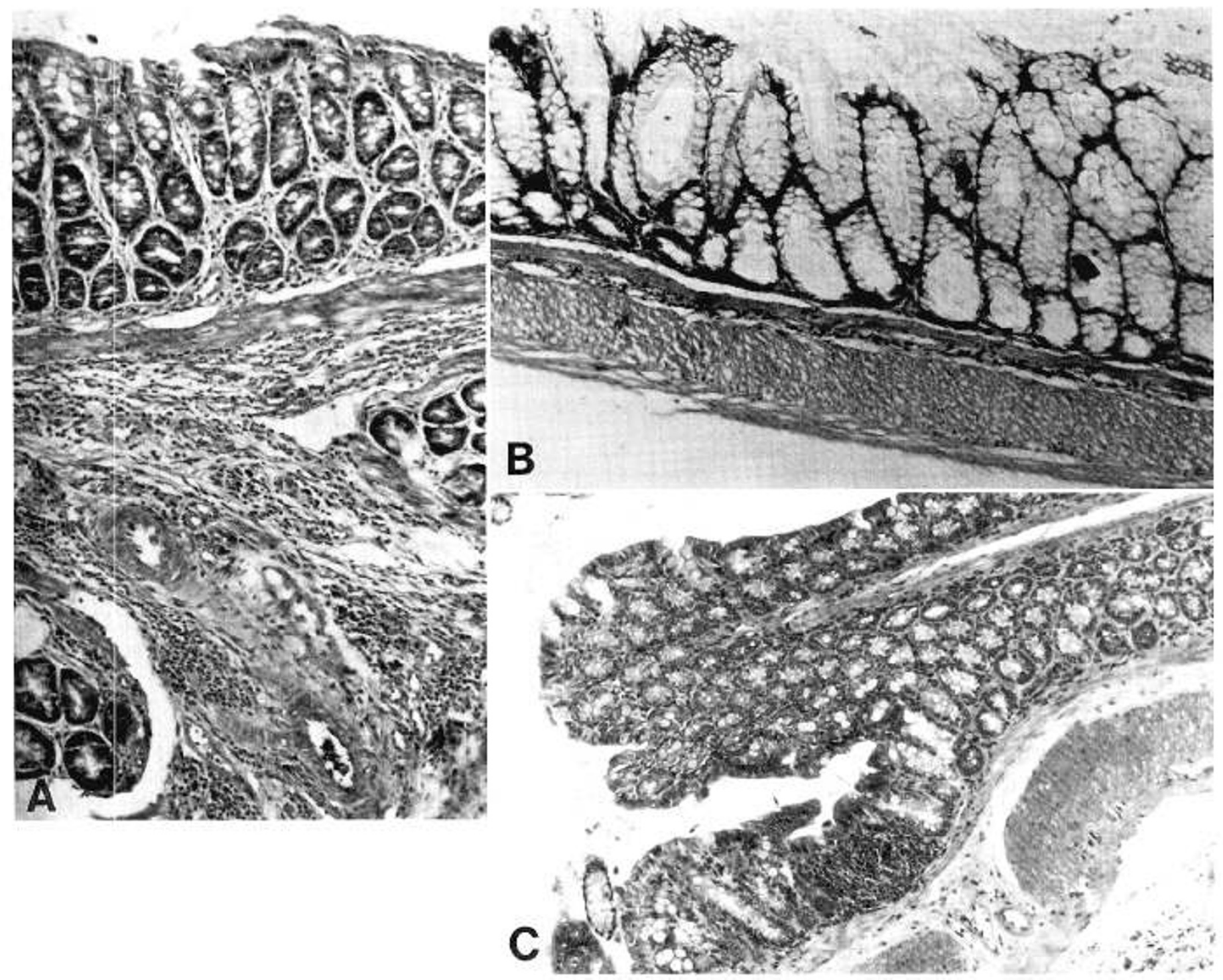

Fig. 1. A, proximal dilated colon of group I mice on hematoxylin and eosin staining showed severe polymorphonuclear leukocyte infiltration, occasional histiocytes, and hemosiderin phagocyte macrophage in the edematous submucosal layer $(\times 125)$. $B$, ganglionic colon from group II mice shows stretched thin muscle coat. The mucosa is flattened with widely distended crypt lumen. Individual goblet cells are filled with mucin and their nuclei are compressed. The mitotic index of goblet cells of this group was extremely low. There is extensive polymorphonuclear infiltration in submucosal layer $(\times 125)$. $C$, control mice (group III) showed well-arranged glandular structure and active goblet cell turnover rate. No inflammatory cell infiltration was observed either in submucosal or lamina propria layer $(\times 125)$.

diameter with impacted feces and the terminal 7 to $13 \mathrm{~mm}$ (mean $10 \mathrm{~mm}$ ) segment of large intestine was markedly constricted and devoid of feces. The spleen was of normal size. The colon from control mice was of normal caliber and contained separate fecal pellets.

Hematoxylin and eosin staining. Surface epithelium and glandular structures were well preserved in all three groups of mice. In the constricted segment of colon in group I and II mice, no ganglion cells were seen in either the myenteric or submucous plexuses but were present in the two plexuses in the more proximal colon normal ganglion cells. Colon from group III mice showed normal frequency and distribution of ganglion cells.

Extensive polymorphonuclear leukocyte infiltration was observed in the edematous submucosal layer and to a lesser degree in the lamina propria of the ganglionic colon in group I mice (Fig. $1 A$ ). There was also round cell infiltration of the lamina propria. Acute splenitis was present in the early death group of mice.

Ganglionic colon from group II mice showed stretched thin muscle tissue making it impossible to differentiate between longitudinal and circular layers (Fig. 1B). Occasionally marked lymphoid hyperplasia was seen compressing the glandular struc- tures. The mucosa was flattened with a widely distended crypt lumen. Individual goblet cells were filled with mucin and their nuclei were compressed. There was significantly increased polymorphonuclear cell infiltration in the submucosal layer. Round cell infiltration was seen around the capillaries in the lamina propria.

Control piebald mice litter mates (group III) showed normal glandular structures and no inflammatory cell infiltration in lamina propria or submucosa (Fig. $1 C$ ). The aganglionic colon from group I and II mice and normal colon from control mice did not show any evidence of inflammatory changes.

Immunocytochemistry. The number of plasma cells of each immunoglobulin isotype infiltrating the lamina propria of the ganglionic colon was significantly increased in group I mice compared to controls $(p<0.001)$ (Fig. 2). Although IgA-producing cells were the dominant plasma cell type in group I mice, IgG and IgM plasma cells were proportionately increased when compared to control mice (Table 1). Each plasma cell type appeared evenly distributed throughout the lamina propria in these mice. Plasma cell infiltration of the lamina propria of the aganglionic noninflammatory portion of the colon was minimal in all mice. 

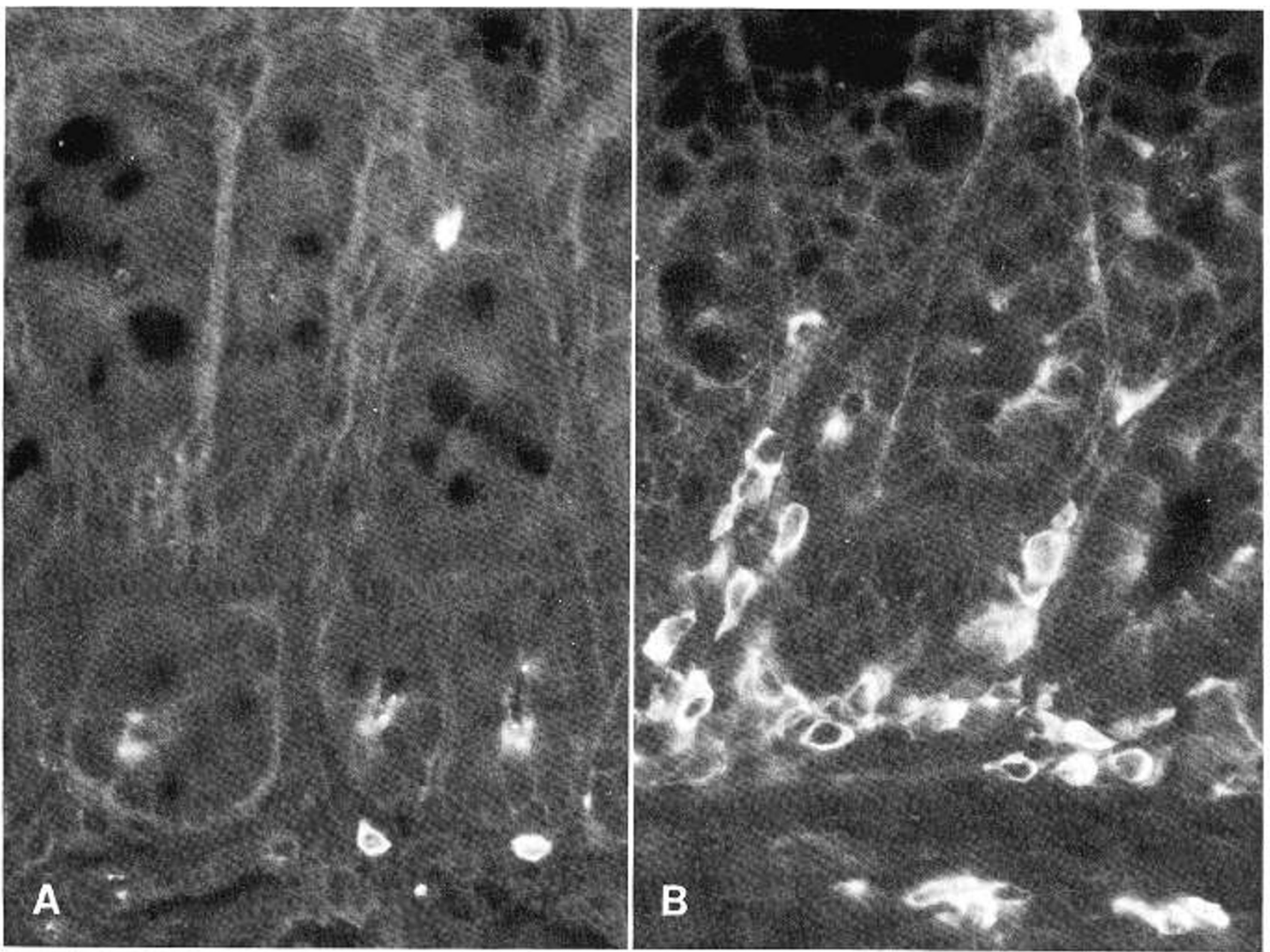

Fig. 2. IgA plasma cell infiltration in lamina propria of piebald lethal mice using immunofluorescence $(\times 400)$. $A$, healthy control mice (group III). $B$, group I mice.

Table 1. Plasma cells in lamina propria of ganglionic colon $\left(\text { mean } \pm 1 \mathrm{SD} / \mathrm{mm}^{2}\right)^{*}$

\begin{tabular}{cccc}
\hline $\begin{array}{c}\text { Plasma cell } \\
\text { isotype }\end{array}$ & $\begin{array}{c}\text { Group I } \\
(n=7)\end{array}$ & $\begin{array}{c}\text { Group II } \\
(n=7)\end{array}$ & $\begin{array}{c}\text { Group III } \\
(n=7)\end{array}$ \\
\hline IgG & $334.3 \pm 113.7$ & $196.9 \pm 55.8$ & $91.0 \pm 38.2$ \\
IgA & $598.0 \pm 146.2$ & $296.1 \pm 65.4$ & $247.1 \pm 48.5$ \\
IgM & $170.7 \pm 47.6$ & $153.3 \pm 69.1$ & $57.0 \pm 12.4$ \\
\hline
\end{tabular}

* IgG I versus III $p<0.001 ;$ II versus III $p<0.001 ;$ I versus II $p<$ 0.005 IgA I versus III $p<0.001$; II versus III NS; I versus II $p<0.001$ IgM I versus III $p<0.001$; II versus III $p<0.001$; I versus II NS. NS ( $p$ $>0.05)$. IgG, IgA, and IgM plasma cells were counted in three visual fields (magnification $\times 25$ ) along the entire colon in 10 serial sections from each mouse.

In group II mice (Table 1) IgG and IgM plasma cells were significantly increased $(p<0.001)$ throughout the lamina propria compared to controls. However, in this group of mice the level of IgA plasma cells was identical to that of control mice. When compared to group I mice the level of IgG plasma cells was significantly lower $(p<0.005)$ in the group II mice and only IgM plasma cell levels were similar in both groups. The distribution of plasma cells in group II mice was confined to the deep layer of lamina propria just above the muscularis mucosa.

\section{DISCUSSION}

This study identified two patterns of local mucosal response in two clinical forms of enterocolitis complicating congenital megacolon in the piebald lethal strain of mice. Both early and late death groups of mice had clear evidence of enterocolitis based on clinical and histological evidence. However, the early death group exhibited a more acute inflammatory response at the time of death as shown by severe polymorphonuclear leukocyte infiltration of submucosa and lamina propria and by the presence of acute splenitis in all mice in this group. The immunochemical investigation of plasma cell isotype distribution clearly distinguished different levels of immunocyte infiltration in the two clinical disease types. Whereas the distribution of plasma cells in the lamina propria in all groups of mice was in the order of IgA, IgG, and IgM only group I mice had significantly increased levels of IgA-containing cells compared to controls. This early death group of mice also had significantly increased levels of IgG and IgM plasma cells compared to group II and control mice. The level of increased immunocyte infiltration in the ganglionic segment of colon involving IgA plasma cells suggests local antigenic stimulation of the colon as the principal pathological event in the early death group of mice.

In contrast the late death group of mice demonstrated a different immunopathological profile from the early death group. The distribution of plasma cell infiltration was confined to the deep layer of lamina propria compared to a diffuse distribution of plasma cells throughout the lamina propria in the early death group of mice. However, the absence of a specific IgA response in the late death group together with the presence of normal spleen histology would suggest that gut-derived infection was not a significant factor in the pathogenesis of this clinical group of mice. It is probable that continued malabsorption in the extended 
ganglionic bowel is the main cause of morbidity in this group of mice.

This study demonstrates that the immunopathology associated with enterocolitis is different in two groups of mice with congenital megacolon. The profile in group I mice is consistent with an underlying local infectious etiology whereas there is no evidence of significant local infection in group II mice. Our findings if applied to humans, would have significant implications for the management of enterocolitis complicating Hirschsprung's disease.

\section{REFERENCES}

1. Nixon HH 1982 Hirschsprung's disease in newborn. In: Holschneider AM (ed) Hirschsprung's Disease. Hippokrates Verlag, Stuttgart, pp 103-113

2. Lillie JG, Crispin AR 1971 Hirschsprung's disease in newborn. Ann Radiol $14: 265-269$

3. Bill AH, Chapman ND 1962 The enterocolitis of Hirschsprung's disease: history and treatment. Am J Surg 103:70-74

4. Fujimoto $\Upsilon$, Puri P 1988 Persistence of enterocolitis folloing diversion of faecal stream in Hirschsprung's disease: a study of mucosal defence mechanism.
Pediatr Surg Int 3:141-146

5. Akkary S, Sahwy E, Kandil W, Handy MH 1981 A histochemical study of the mucosubstances of the colon in cases of Hirschsprung's disease with and without enterocolitis. J Pediatr Surg 16:664-668

6. Berry CL, Fraser GC 1968 The experimental production of colitis in the rabbit with particular reference to Hirschsprung's disease. J Pediatr Surg 3:36-42

7. Lloyd-Still JD, Dermers LM 1978 Hirschsprung's enterocolitis: prostaglandins and response to cholestyramine. J Pediatr Surg 13:417-418

8. Thomas DFM, Fernie DS, Bayston R, Spitz L, Nixon HH 1986 Enterocolitis in Hirschsprung's ciisease: a controlled study of the etiologic role of clostridium difficile. J Pediatr Surg 21:22-25

9. Webster W 1974 Aganglionic megacolon in piebald-lethal mice. Arch Pathol 97:111-117

10. Richardson J 1975 Pharmacologic studies of Hirschsprung's disease on a murine model. J Pediatr Surg 10:875-884

11. Bu Lock A, Vaillant C, Dockray GJ 1984 Selective depletion of substance Pimmunoreactive neurons in the transitional zone of the colon in piebald lethal mice. Neurochem Int 6:55-61.

12. Fujimoto $T 1988$ Natural history and pathophysiology of enterocolitis in the piebald lethal mouse model of Hirschsprung's disease. J Pediatr Surg 23:237242

13. Brandtzaeg P, Rognum TO 1984 Evaluation of nine different fixatives. 1. Preservation of immunoglobulin isotypes, $\mathrm{J}$ chain, and secretory component in human tissue. Pathol Res Pract 179:250-266 